\title{
Repeated measures regression mixture models
}

\section{Minjung Kim ${ }^{1} \cdot$ M. Lee Van Horn ${ }^{2} \cdot$ Thomas Jaki $^{3}$ • Jeroen Vermunt ${ }^{4} \cdot$ Daniel Feaster $^{5} \cdot$ Kenneth L. Lichstein $^{6}$. Daniel J. Taylor ${ }^{7} \cdot$ Brant W. Riedel $^{8} \cdot$ Andrew J. Bush ${ }^{9}$}

Published online: 31 May 2019

(C) The Psychonomic Society, Inc. 2019

\begin{abstract}
Regression mixture models are one increasingly utilized approach for developing theories about and exploring the heterogeneity of effects. In this study we aimed to extend the current use of regression mixtures to a repeated regression mixture method when repeated measures, such as diary-type and experience-sampling method, data are available. We hypothesized that additional information borrowed from the repeated measures would improve the model performance, in terms of class enumeration and accuracy of the parameter estimates. We specifically compared three types of model specifications in regression mixtures: (a) traditional single-outcome model; (b) repeated measures models with three, five, and seven measures; and (c) a single-outcome model with the average of seven repeated measures. The results showed that the repeated measures regression mixture models substantially outperformed the traditional and average single-outcome models in class enumeration, with less bias in the parameter estimates. For sample size, whereas prior recommendations have suggested that regression mixtures require samples of well over 1,000 participants, even for classes at a large distance from each other (classes with regression weights of .20 vs. .70), the present repeated measures regression mixture models allow for samples as low as 200 participants with an increased number (i.e., seven) of repeated measures. We also demonstrate an application of the proposed repeated measures approach using data from the Sleep Research Project. Implications and limitations of the study are discussed.
\end{abstract}

Keywords Regression mixture models $\cdot$ Sample size $\cdot$ Repeated measures $\cdot$ Heterogeneous effects

Regression mixture models are a novel approach to identify heterogeneous effects. These models detect two or more subpopulations, which differ in the effect of a predictor on an outcome, using a latent class variable instead of the a priori moderator(s). For example, regression mixture models were used to find a differential susceptibility to the effects of a child's temperament on the mother-child interaction style (Lee, 2013), in which they found three latent classes with varying associations between the child's temperament and mother-child interactions (i.e., nonsusceptible class,

Minjung Kim

Kim.7144@osu.edu

M. Lee Van Horn

mlvh@unm.edu

1 Department of Educational Studies, Ohio State University, Columbus, OH, USA

2 Department of Individual, Family, and Community Education, University of New Mexico, Albuquerque, NM, USA

3 Department of Mathematics and Statistics, Lancaster University, Lancaster, UK susceptible-high class, and susceptible-low class). In another study, regression mixture approach was used to understand the differential effects of depressive symptoms and neuroticism on cognitive complaints in older adults (Kliegel \& Zimprich, 2005). Given the exploratory nature of this approach, regression mixture models have become increasingly popular in the last decade (Dyer, Pleck, \& McBride, 2012; Lanza, Cooper, \& Bray, 2014; Lanza, Kugler, \& Mathur, 2011; McDonald et al., 2016; Schmiege \& Bryan, 2016; Silinskas et al., 2013; Silinskas et al., 2016).

4 Department of Methodology and Statistics, Tilburg University, Tilburg, The Netherlands

5 Department of Public Health Sciences, Division of Biostatistics, University of Miami, Miami, FL, USA

6 Department of Psychology, University of Alabama, Tuscaloosa, AL, USA

7 Department of Psychology, University of North Texas, Denton, TX, USA

8 Shelby County Schools, Memphis, TN, USA

9 Department of Preventive Medicine, University of Tennessee, Knoxville, TN, USA 
The present study introduces the use of regression mixture models with repeated measures. One of the commonly used mixture approaches to repeated measures is a growth mixture model (B. Muthén, 2004; B. Muthén \& Muthén, 2000). Growth mixture models have been increasingly popular and applied in a wide range of fields including health, educational, and psychological studies. The main purpose of growth mixture models is to identify heterogeneity in growth trajectories assuming that the data are from a mixture of subpopulations of individuals with different growth trajectories. Growth mixture models identify these subpopulations by estimating classspecific growth parameters (e.g., intercept, change of rate, variance). Under the framework of multilevel modeling (i.e., repeated measures are nested within individuals), random-effects mixture models (Ng, McLachlan, Wang, Ben-Tovim Jones, \& Ng, 2006; Xu \& Hedeker, 2001) have been also widely employed to unfold the possible subpopulation heterogeneity. Similar to the growth mixture models, the main interest in the use of random-effect mixture models has typically been in determining heterogeneity in the growth trajectory of repeated outcomes over time (Kohli, Harring, \& Zopluoglu, 2016; Yau, Lee, \& Ng, 2003), where the latent classes are identified on the basis of the fixed and random effects at each time point.

Although finite mixtures have been used extensively for examining heterogeneity in longitudinal trajectories (Bauer \& Curran, 2003; Jung \& Wickrama, 2008; Nylund, Asparouhov, \& Muthén, 2007), our purpose in using repeated measures regression mixtures was quite different. Rather than focusing on trends over time, repeated measures are collected to increase the amount of information available about the outcome. Obvious uses of this approach are situations in which repeated measures data are available but the longitudinal trends are not of interest, but are considered nuisance parameters. Examples in which this may be the case include daily diary data (such measures may include sleep quality, substance use, daily mood, or affect) and data collected by loggers, such as accelerometry measures of physical activity or logs of blood pressure. In these cases, researchers are interested in the true state of a behavior, but they need to measure it multiple times to ensure they are capturing it with accuracy. Current state-of-the-art practice with such data is to either average across the multiple observations or create a latent factor from the multiple observations, which factors out the error and get a closer approximation of the true score. In repeated measures regression mixture models, the primary interest is in how such a factor or construct varies in its relation with other constructs, while time is considered a nuisance rather than a factor of interest to explore. We propose that repeated measures regression mixtures can benefit from the information gained when using all available data rather than a summary score.

Regression mixture models are a specific type of finite mixtures, which examines heterogeneity in the regression of an outcome on predictor(s). Heterogeneity in effects is assessed by differences in the regression weights (e.g., intercept, slope), which connect predictor(s) to outcome(s) across latent classes. Whereas a single set of average regression weights are estimated for all subjects in a traditional regression model, two or more sets of average regression weights are estimated for the corresponding number of latent classes in a regression mixture model. A general regression mixture model for a single outcome is:

$y_{i \mid X, k}=\beta_{0 k}+\sum_{p=1}^{P} \beta_{p k} x_{i p}+\varepsilon_{i k}, \quad \varepsilon_{i k}, \sim N\left(0, \sigma_{k}^{2}\right)$,

where $y_{i \mid X, k}$ represents the observed value of $y$ for subject $i$ in a specific class $k$ given predictor $X, \beta_{0 k}$ captures the class-specific intercept coefficient, and heterogeneity in the effects of each predictor is captured by $\beta_{p k}$, which represents the class-specific slope of the corresponding predictor, $X_{p}$. In line with traditional linear models, the errors within each class, $\varepsilon_{i k}$, are assumed to be normally distributed with a mean of zero and a class-specific variance of $\sigma^{2}{ }_{k}$. Each observation (subject) is assigned to a latent class using a multinomial equation as a function of the overall latent class probabilities:

$\operatorname{Pr}\left(\mathrm{c}_{\mathrm{i}}=\mathrm{k} \mid \mathbf{z}_{i}\right)=\frac{\exp \left(\alpha_{\mathrm{k}}+\sum_{\mathrm{q}=1}^{\mathrm{Q}} \gamma_{\mathrm{qk}} \mathrm{z}_{\mathrm{iq}}\right)}{\sum_{\mathrm{s}=1}^{\mathrm{K}} \exp \left(\alpha_{\mathrm{s}}+\sum_{\mathrm{q}=1}^{\mathrm{Q}} \gamma_{\mathrm{qs}} \mathrm{z}_{\mathrm{iq}}\right)}$,

where $\alpha_{k}$ denotes the log odds of being in class $k$ versus the reference class when all latent class predictors, $z_{q}(q=$ $1, \ldots, Q)$, equal zero. The potential difference in the predictor variable $X_{p}$ between the latent classes can be taken into account by allowing the $C$ on $X$ path in the model (Lamont, Vermunt, \& Van Horn, 2016). Figure 1 presents the example diagram of a regression mixture model with a single outcome $(y)$, a single predictor $(x)$, and where $(c)$ represents the unobserved (latent) class membership.

When there are additional data for the outcome measure, we incorporated the framework of latent growth models (LGMs; Duncan, Duncan, \& Strycker, 2013) into the regression mixture models. Although the present method can be extended to more complex models with additional time functions (e.g., linear or quadratic growth models), we focused on building a random-intercept-only model for the repeated measures, in which interindividual and intra-individual differences are modeled adequately. We specifically focused on detecting the heterogeneity in the effect of predictor(s) on the latent intercept. Adding additional latent factors for the time function (e.g., linear, quadratic slope factors) can be also considered after conducting more exploratory analyses, such as visual inspection using graphical function in statistical software.

Figure 2 represents the regression mixture model with three repeated measures of data. The single-outcome 


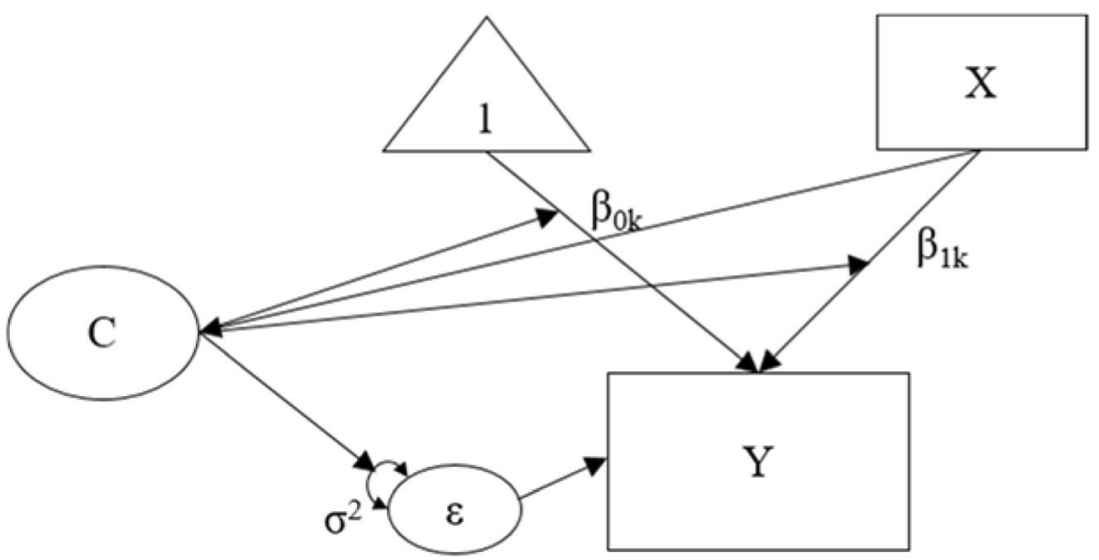

Fig. 1 Regression mixture model with a single outcome and a predictor

regression mixture model can be extended for repeated measures, as

$\mathbf{Y}_{\mathbf{i} \mid \mathbf{X}, \mathbf{K}}=\boldsymbol{\tau}+\boldsymbol{\Lambda} \boldsymbol{\eta}_{\boldsymbol{i k | X}}+\varepsilon_{t i \boldsymbol{k}}, \quad \varepsilon_{t i k}, \sim N\left(0, \sigma_{t k}^{2}\right)$,

where $\mathbf{Y}_{\boldsymbol{i}}$ refers to a vector of outcome variables $(t \times 1$, where $t$ is the number of repeated measures from $i$ individuals), $\tau$ refers to a vector of intercepts of $y s$ ( $t \times 1$; typically fixed to zero for model identification purpose), $\boldsymbol{\Lambda}$ relates the $t$ observed repeated measures from the $i$ individuals to the intercept $\left(\boldsymbol{\eta}_{\boldsymbol{i k}}\right)$ for each latent class given the predictor $X_{p}$, and $\varepsilon_{t i k}$ represents the class-specific measurement errors of the repeated response variables $(t \times 1$; a vector of within-subjects residual variance of $y s$ for each class). In the simplest case, the $\Lambda$ vector is fixed to be 1 at all measurement points, to represent the average value of repeated outcomes across times, and the variance-covariance matrix $\sum$ is a diagonal matrix with all diagonal elements fixed to be equal:

$\operatorname{Var}\left[\begin{array}{l}\varepsilon_{1} \\ \varepsilon_{2} \\ \varepsilon_{3}\end{array}\right]=\left[\begin{array}{ccc}\sigma_{1 k}^{2} & 0 & 0 \\ 0 & \sigma_{1 k}^{2} & 0 \\ 0 & 0 & \sigma_{1 k}^{2}\end{array}\right]$,

so that the residual variances are constant over time and uncorrelated for each pair of time measures (this is often called the identity [ID] error structure). More complex error variance

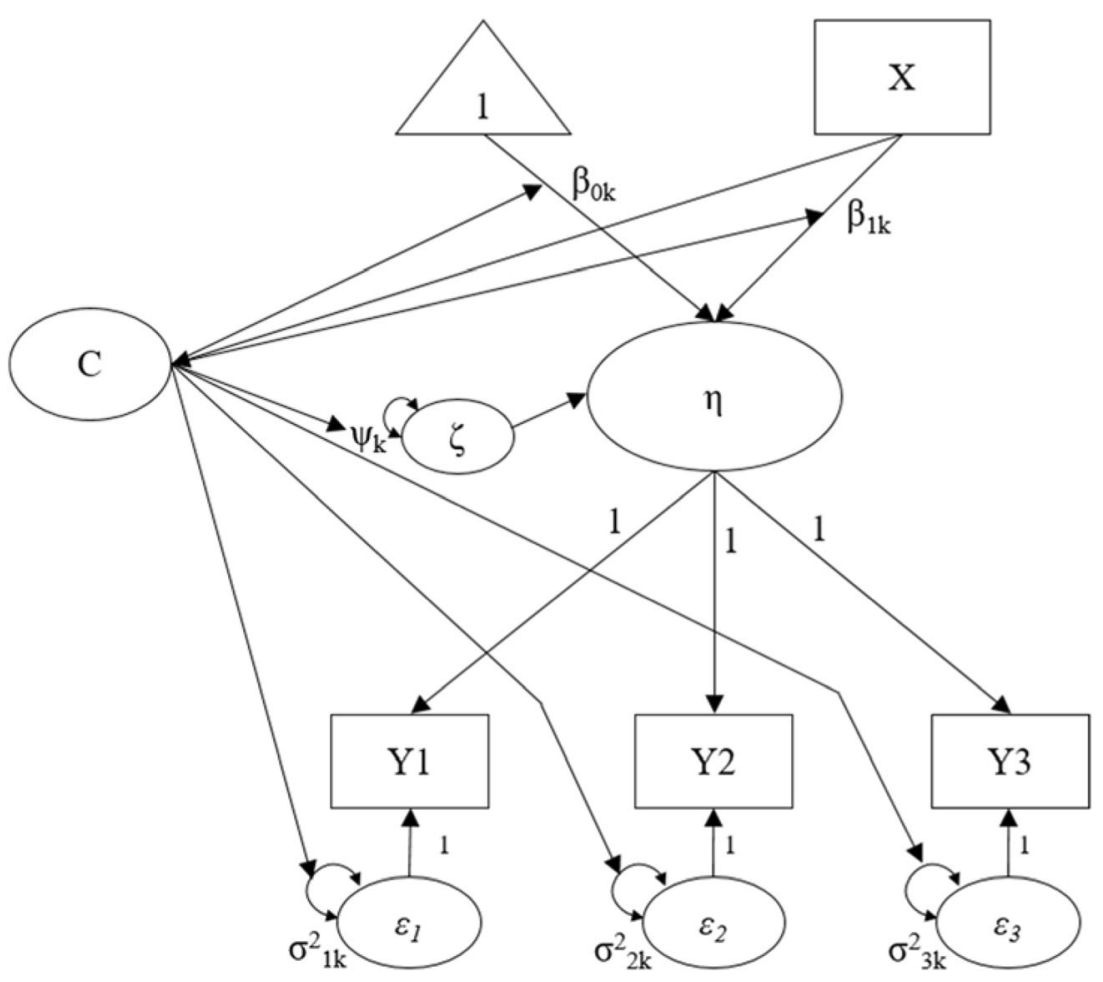

Fig. 2 Regression mixture model with three repeated measures 
structures (e.g., compound symmetry, auto-regressive, bandeddiagonal, etc.) could be considered. For the present study, however, we use the simplest ID structure for specifying the repeated measures regression mixture model as the method has limited value if it does not perform well under the simplest model. These restrictions presume that the focus of the model is on predicting the outcome equally across all repeated measures and that the residual variances across time points have no systematic correlations.

Given the measurement model for $\boldsymbol{\eta}$ in Eq. 3, the focus of the regression mixture is on the prediction of $\boldsymbol{\eta}$ as a function of the class-specific effects of $p$ covariates. Thus, $\boldsymbol{\eta}$ becomes the outcome in Eq. 1.

$\eta_{i k \mid X}=\beta_{0 k}+\sum_{p=1}^{P} \beta_{p k} x_{i p}+\zeta_{i k}, \quad \zeta_{i k} \sim N\left(0, \psi_{k}\right)$.

In Eq. $4, \beta_{0 k}$ represents the mean of the repeated outcomes for each class when the predictor $x_{i p}$ is zero, $\beta_{p k}$ represents the class-specific regression coefficients of $x_{p}$, as before, and $\zeta$ represents the deviation of the corresponding individual values from the mean estimates of the latent intercept factor. Along with the single latent intercept factor, there is one between-subjects variance component (i.e., $\psi_{k}$ ), which captures the average deviation from the grand mean (i.e., mean of the repeated measures across all subjects) to the individual mean for the intercept factor.

Although the present model is formulated under the LGM framework, it can be reproduced under the multilevel modeling (MLM) framework, given the equivalence of the two model frameworks (Grimm, Ram, \& Estabrook, 2017). When the model is reproduced in MLM, the two-level model (i.e., repeated measures are nested within individuals) can be employed by having the total variance decomposed into $\psi_{k}$ (i.e., between-subjects variance) and $\sigma_{t k}^{2}$ (i.e., within-subjects variance). In the latent-variable framework, this is achieved by having separate (correlated) variances across all of the measurement occasions.

\section{Study aims}

In this study we aimed (1) to evaluate the performance of repeated measures regression mixtures and (2) to demonstrate the use of this model to examine the differential effects of stress on sleep diary in a sample obtained from a prior study. First, performance of the repeated measures regression mixture model was examined through Monte Carlo simulations in which we varied the measurement error variance of the outcome across repeated measures, the number of repeated measures, and the sample size. To understand the usefulness of this approach in practice, we also compared the results of the repeated measures regression mixtures to two cases. The first case was a standard regression mixture in which only one observation of the outcome was collected. This shows the potential benefit of collecting additional outcome data when planning a study, which will use regression mixtures. The second case compared the repeated measures regression mixture to the results that would be obtained if all repeated measures were available but the analysis conducted was a standard regression mixture, with the outcome being the average across all time points. The purpose of this second case was to examine whether the benefits of using repeated measures data are due to simultaneously modeling multiple outcomes or to the increased reliability from having additional data points. If the two approaches (i.e., the repeated measures regression mixture approach and the single outcome with the average score regression mixture approach) were to show no difference in improvement over the traditional single-outcome approach, we would conclude that the benefits were due solely to the increased reliability but not to simultaneously modeling multiple outcomes.

The outcomes in this study were both the latent class enumeration (measured as the proportion of simulations that selected the correct number of classes) and the bias and efficiency of the parameter estimates. We hypothesized that both outcomes would be improved as the number of measurements and sample size increased. We also hypothesized that the benefit of having multiple outcomes would be greater when the measurement error variance was larger, primarily due to jointly modeling these repeated measures outcomes. The reason for this was that the regression mixture model with a single outcome relies strongly on assumptions about the conditional distribution of that outcome for parameter estimation (Van Horn et al., 2015); with multiple outcomes, estimation is informed by the joint distribution of all outcomes, which contains more information than does the univariate distribution.

We then demonstrated the use of repeated measures regression to identify qualitatively different groups of people who differed in the effects of cognitive and behavioral symptoms of depression on sleep efficiency. Sleep efficiency was measured using daily diaries, an example of the type of application that might benefit from repeated measures regression mixtures. We believe that this proposed method can be especially beneficial for clinical research, which often includes relatively small sample sizes and often has an inherent interest in individual differences.

\section{Method}

\section{Data generation}

In the first part of this study, we used Monte Carlo simulations to examine the performance of the repeated measures regression mixture model. Data were generated using R (R Core Team, 2017) for a two-class model as the true population model. Each class was defined by an intercept and slope (i.e., Class 1: $\beta_{00}=0.0$ and $\beta_{10}=0.20$ or 0.40 for the small 
or medium effect class, respectively; Class $2: \beta_{01}=0.50$ and $\beta_{11}=0.70$ for the large effect class). The regression weights for Class 1 were set to be either small $\left(\beta_{10}=0.20\right)$ or medium $\left(\beta_{10}=0.40\right)$, so that the distances from Class $2\left(\beta_{11}=0.70\right)$ were set to be either large or small, respectively. We anticipated that the regression mixture models would perform poorly when the Class 1 effect size was medium, given the reduced class separation from Class 2 (i.e., 0.40 vs. 0.70). We used the current setting to generate the two latent classes because we believe that if regression mixtures are to be useful for finding individual differences, they should be able to detect a difference of at least this size (Kim, Vermunt, Bakk, Jaki, \& Van Horn, 2016; Van Horn et al., 2015). Although the entropy is regarded as a model fit index to show the accuracy of class assignment in general finite mixture models, it is not necessarily indicative of goodness of fit in regression mixture models. Therefore, the entropy was not considered to be a design factor for our simulation study.

We generated seven repeated measures outcome for each condition, because collecting daily data on a weekly basis is common in much applied research. Additionally, we varied the proportion of measurement error variance, $\sigma_{1 k}^{2}(1 \%$, $10 \%, 20 \%$, or $40 \%$ of the total variance ${ }^{1}$ ), ranging from minimal (1\%) to substantial (40\%). The reason we included the minimal measurement error condition was that we often see that there are variables that rarely change over time (e.g., daily body mass index). When the measures are extremely stable and not changed, we expect that the benefits of having additional time points will be much smaller than when there is some extent of measurement errors. These simulations included four different sample size conditions, from 200 as a small sample size condition, to 3,000 as a large sample size condition. Although previous simulation studies had shown that 3,000 is an adequate number for detecting the differential effect with a large effect size difference, many applied studies have employed the regression mixture approach with much smaller sample sizes (Dyer et al., 2012; McDonald et al., 2016; Wong, Owen, \& Shea, 2012). Therefore, we included 200 as the smallest sample size condition in the present study. Sample sizes for the two classes were balanced, having $50 \%$ of the total samples assigned to each class $\left(\alpha_{k}=0.0\right)$. The predictor $X$ was generated from a standard normal distribution with a mean of 0 and a standard deviation of 1 . Although it is important to model the potential mean difference in $X$ between classes, we did not include this additional condition in the present simulations.

For data generation, there were a total of 32 separate conditions ( 2 effect size distance $\times 4$ error variances $\times 4$ sample sizes), with 500 replications used for each condition. The total amount of variance, $\beta_{1 k}^{2}+\sigma_{1 k}^{2}+\psi_{k}$, for each outcome was set to be 1 for all conditions. The following equations were used to generate the seven-time-point repeated measures data, by varying the four conditions of measurement variance:

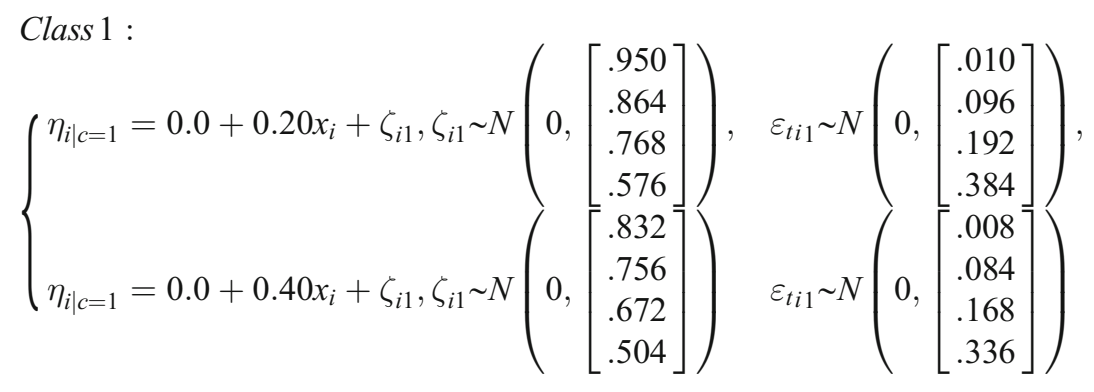

Class 2 :

$$
\eta_{i \mid c=2}=0.5+0.70 x_{i}+\zeta_{i 2}, \zeta_{i 2} \sim N\left(0\left[\begin{array}{l}
.505 \\
.459 \\
.408 \\
.306
\end{array}\right]\right) \varepsilon_{t i 2} \sim N\left(0,\left[\begin{array}{l}
.005 \\
.054 \\
.102 \\
.204
\end{array}\right]\right),
$$

where $\zeta$ and $\varepsilon$ are assumed to be normally distributed. The simplest variance-covariance $(\mathrm{V}-\mathrm{CV})$ structure was adopted

\footnotetext{
${ }^{1}$ For $\psi_{\mathrm{k}}$, population values were set to be $99 \%, 90 \%, 80 \%$, and $60 \%$, accordingly.

${ }^{2}$ This eventually makes the compound symmetry for the repeated outcome measures, given the shared variance through the intercept variance, $\psi$.
}

for the within-subjects residual V-CV (i.e., identity structure), ${ }^{2}$ because we specifically aimed to use this approach for the repeated measures collected in a short period of time; we did not have a specific hypothesis for the error variance structure. Although it has been shown that the results of regression mixture models are affected by the error variance 
distribution (Van Horn et al., 2012), examining nonnormal errors was beyond the scope of the present study.

\section{Data analysis}

Before analyzing the regression mixture models, a multiple regression model with a predictor $X$ and a binary indicator of class membership, $Z$ (i.e., $y_{i}=\beta_{0}+\beta_{1} X+\beta_{2} Z+\beta_{3} X^{*} Z+e_{i}$ ), was analyzed to confirm the data generation process. We used Mplus7.3 (L. K. Muthén \& Muthén, 1998-2012) employing the maximum likelihood estimator with robust standard errors (ESTIMATOR $=$ MLR) to analyze each dataset. Five types of regression mixture models were analyzed for each dataset: (A) a traditional regression mixture model with a single outcome measure (one of the seven repeated measures), (B) a threerepeated-measures regression mixture model, (C) a fiverepeated-measures regression mixture model, (D) a sevenrepeated-measures regression mixture model, and (E) a model of the average of all seven-repeated-measures in a regression mixture model. For Models A, B, and C, the first one, three, and five variable(s) of the seven generated outcomes were used for the data analysis, respectively. For Model E, the average score of the seven repeated measures was used as a single outcome in a regression mixture analysis.

For all five specifications of regression mixture models, a series of analyses was conducted for each simulation, varying the number of latent classes. First, a one-class model, which is a traditional regression model with no mixture, is analyzed. The results were compared with the next model, having one more class (two-class model), using the Bayesian information criterion (BIC; Schwarz, 1978) and the sample-size-adjusted BIC (ABIC; Sclove, 1987). Finally, a three-class model was estimated for each simulated dataset to assess the probability of finding three rather than two classes. The number of simulations finding the correct number of classes (i.e., two classes) will be reported in the class enumeration results. Accuracy of the parameter estimates was assessed using absolute bias in the parameter estimates to the population values, based on the following equation:

$B(\overline{\hat{\beta}})=\overline{\hat{\beta}_{\text {est }}}-\beta_{\text {pop }}$.

In addition, the coverage rate for the true population value is also reported. More specifically, the coverage is coded as 1 if the true population value was included in the $95 \%$ confidence interval (CI), or 0 if it was outside the $95 \%$ CI. Only replications selecting the true two-class model using the BIC are included for calculating the bias as well as the coverage rate of the estimated parameters. ${ }^{3}$ To account for the problem of switched labels in the simulations (McLachlan \& Peel,

\footnotetext{
$\overline{3}$ The full results, including the parameter estimates for all simulation conditions, are available from the first author.
}

2000; Sperrin, Jaki, \& Wit, 2010), we first sorted the two classes by the slope coefficients so that the smaller effect size class was always labeled Class 1, and the larger effect size class was Class 2.

\section{Results}

\section{Class enumeration}

All one-class models converged, but the convergence rates for the two-class and three-class models ranged from $99.2 \%$ and $96.4 \%$, respectively, to $100 \%$, depending on the sample size. Table 1 presents the class enumeration results using the BIC. ${ }^{4}$ Percentages of simulations detecting the true two classes are shown for each condition analyzed. Overall, the probability of selecting the correct two-class model increases as sample size increases across all conditions, as expected.

Adding additional time points The primary question in the present study was whether the class enumeration result would be improved by introducing the repeated measures into the regression mixture model. This was assessed by comparing the results of the regular (single-outcome) regression mixture (Model A) to the models with multiple repeated measures (Models B to D). As is shown in Table 1, increasing the number of repeated measures in the regression mixture model substantially improves the probability of detecting the correct two classes, using both the BIC and the ABIC. The average detection rate of $^{5} 28.9 \%$ for Model A using the BIC under the large distance condition is increased to $51.3 \%, 71.2 \%$, and $82.8 \%$ for Models B, C, and D, respectively. Similarly, the detection rate under the small distance condition is gradually increased from $5.7 \%$ (Model A) to $52.9 \%$ (Model D) on average. Although the ABIC is shown to be less sensitive to the different model specifications, the detection rate using the ABIC steadily increases as well by adding more repeated measures into the model. ${ }^{6}$

As we hypothesized, the impact of adding additional time points on the class enumeration is greater under the small sample size condition. For a sample size of 200 with the large distance condition, the probability of selecting the two-class model using the BIC is substantially increased from Model A (average of $7.9 \%$ ) to Model D (average of 52.3\%) across different measurement error variance conditions. When the sample size is 500, Model D (seven repeated measures) selects the correct two classes in above $96 \%$ of simulations using the

\footnotetext{
${ }^{4}$ Class enumeration results using the $\mathrm{ABIC}$ can be requested from the first author.

${ }^{5}$ This is calculated using the sample size of 200,500 , and 1,000 , excluding 3,000 , across all conditions of measurement error variance.

${ }^{6}$ The results of the detection rate using the ABIC can be obtained by the request to the first author.
} 
Table 1 Class enumeration results for percentages of simulations selecting the true two-class model by the BIC

\begin{tabular}{|c|c|c|c|c|c|c|c|}
\hline Effect Size Distance & Measurement Error & Sample Size & (A) One-Time & (B) Three-Time & (C) Five-Time & (D) Seven-Time & (E) Seven-Time Average \\
\hline Large & 1 & 200 & 8.2 & 8.4 & 23.2 & 46.4 & 7.0 \\
\hline \multirow[t]{16}{*}{$(.20 \mathrm{vs} .70)$} & & 500 & 16.4 & 38.8 & 79.2 & 96.4 & 18.0 \\
\hline & & 1,000 & 63.0 & 90.0 & 99.2 & 99.6 & 62.6 \\
\hline & & 3,000 & 99.6 & 99.6 & 99.8 & 99.8 & 100.0 \\
\hline & 10 & 200 & 7.4 & 9.2 & 25.6 & 48.8 & 7.8 \\
\hline & & 500 & 21.4 & 44.0 & 84.8 & 96.4 & 25.2 \\
\hline & & 1,000 & 58.0 & 92.0 & 99.0 & 99.2 & 69.6 \\
\hline & & 3,000 & 99.6 & 99.2 & 99.2 & 99.4 & 99.6 \\
\hline & 20 & 200 & 9.0 & 10.4 & 26.4 & 51.8 & 10.2 \\
\hline & & 500 & 21.2 & 52.6 & 86.6 & 96.4 & 35.6 \\
\hline & & 1,000 & 58.4 & 93.2 & 99.4 & 99.8 & 75.4 \\
\hline & & 3,000 & 99.8 & 99.4 & 99.2 & 99.6 & 99.6 \\
\hline & 40 & 200 & 7.0 & 17.4 & 39.0 & 62.0 & 16.2 \\
\hline & & 500 & 18.8 & 61.8 & 92.4 & 98.2 & 49.4 \\
\hline & & 1,000 & 58.2 & 98.0 & 99.2 & 98.6 & 92.0 \\
\hline & & 3,000 & 99.6 & 99.2 & 99.4 & 99.4 & 100.0 \\
\hline & Average rate ${ }^{\mathrm{a}}$ & & 28.9 & 51.3 & 71.2 & 82.8 & 39.1 \\
\hline Small & 1 & 200 & 3.6 & 1.6 & 5.8 & 10.6 & 4.0 \\
\hline \multirow[t]{16}{*}{$(.40$ vs . 70$)$} & & 500 & 5.0 & 6.4 & 16.2 & 44.0 & 5.2 \\
\hline & & 1,000 & 8.2 & 22.4 & 62.8 & 92.2 & 8.6 \\
\hline & & 3,000 & 77.2 & 98.6 & 99.8 & 99.4 & 78.2 \\
\hline & 10 & 200 & 2.8 & 1.6 & 3.6 & 9.6 & 3.8 \\
\hline & & 500 & 2.8 & 5.6 & 19.6 & 50.0 & 4.8 \\
\hline & & 1,000 & 11.2 & 31.2 & 69.8 & 92.6 & 15.4 \\
\hline & & 3,000 & 75.2 & 97.8 & 98.8 & 99.2 & 81.8 \\
\hline & 20 & 200 & 3.0 & 1.8 & 6.2 & 9.6 & 4.6 \\
\hline & & 500 & 4.0 & 8.4 & 26.0 & 52.0 & 7.4 \\
\hline & & 1,000 & 11.8 & 33.2 & 72.4 & 92.8 & 17.2 \\
\hline & & 3,000 & 78.6 & 98.8 & 98.6 & 99.0 & 92.4 \\
\hline & 40 & 200 & 3.0 & 3.2 & 8.8 & 18.0 & 5.2 \\
\hline & & 500 & 4.4 & 10.6 & 32.6 & 65.4 & 7.4 \\
\hline & & 1,000 & 8.2 & 47.0 & 85.6 & 97.6 & 32.6 \\
\hline & & 3,000 & 78.8 & 97.8 & 99.0 & 99.0 & 98.6 \\
\hline & Average rate ${ }^{a}$ & & 5.7 & 14.4 & 34.1 & 52.9 & 9.7 \\
\hline
\end{tabular}

${ }^{a}$ Average rate is calculated using the sample size of 200,500, and 1,000 excluding 3,000 across all measurement error variance conditions

BIC, whereas it is still below $20 \%$ for traditional regression mixtures (Model A) on average, across all measurement error variance conditions. The class enumeration results for using the repeated measures are very promising when the sample size is 1,000 under the large distance condition. The detection rate is $90 \%$ or above for using three or more repeated measures in the model, regardless of the degree of measurement error variance. Although the same pattern is observed for the small distance condition, more repeated measures (at least five or seven) are required in order to achieve a power greater than .80. In sum, under the condition of small to moderately large sample sizes, introducing repeated measures into the regression mixtures greatly increases the utility of the method to detect the potential differential effects.

Using the average value of the repeated measures Next, we compared the class enumeration results of Model A and Model D to the results of an average composite model of the seven repeated measures (Model E). It is a common approach to use a composite variable of the repeated measures, such as mean and sum scores, when the measures are expected to be stable with no systematic change over time. It is reasonable to expect that the average score represents the data better than the one-time measure at any point when the measurement error 
exists. Although there is an overall positive effect for using the average score over the single-time-point measure on the class enumeration results, the use of repeated measures (Models B, $\mathrm{C}$, and D) outperforms the use of the average score in regression mixtures, using both the BIC and the ABIC.

Although the detection rate is slightly increased from Model A to Model E by using the average score of the seven repeated measures, no major benefit was shown for using the composite variable in regression mixture results. On the other hand, as compared to Model E, using the original seven repeated measure variables in regression mixture models (Model D) substantially improves the class enumeration results. Under the condition of a large distance between the two effect sizes, Table 1 shows that the average detection rate using the BIC is $28.9 \%$ for Model A, and 39.1\% for Model E, whereas it is drastically increased to $82.8 \%$ for Model D. Similarly, the average detection rate is increased from $5.7 \%$ (Model A) and 9.7\% (Model E) to 52.9\% (Model D) by employing the repeated measures in the regression mixtures under the small distance condition.

\section{Measurement error variance in repeated regression mixtures}

As is shown in Table 1, the degree of measurement error variance has no significant impact on the class enumeration results for the single-outcome measure (Model A), other than the random sampling error. Given that the intercept variance and the residual variance are combined as one total residual variance component for Model A, it is not surprising that the class enumeration results are consistent under the same conditions, with a total variance of 1 .

The interesting finding is that the impact of introducing the repeated measures into the regression mixtures is much greater when the measurement error variance gets larger. When the ratio of error variance is only $1 \%$, the average rate for detecting the true two classes is increased from $29.2 \%$ to $45.7 \%$, from Model A to Model B, respectively. As compared to the $1 \%$ condition, when the measurement error variance is increased to be $10 \%, 20 \%$, or $40 \%$ of the total residual variance, the average detection rates increase to $48.4 \%, 52.1 \%$, and $59.1 \%$, respectively. That is, the impact of having more information from the repeated measures is greater when the measurement reliability is more problematic, given the increased error variance. This can be understood as the greater shared variance across the repeated measures (higher ratio of intercept variance to the total variance ${ }^{7}$ ) adding less information for estimating the parameters. In the extreme scenario, when the ratio of the intercept variance to the total variance is 1 , the repeated measures are perfectly correlated, so they are redundant information. For Model C with a small or moderate sample size, the same pattern is shown, that the detection rate

\footnotetext{
${ }^{7}$ This refers to the intraclass correlation (ICC), in the framework of multilevel modeling.
}

steadily increases as the measurement error variance increases.

Distance in the effect size difference between classes Under the condition of a large distance in effect sizes between the two classes (.20 vs. .70 condition), when the sample size is large $(n=3,000)$, two classes are successfully detected in most simulations, regardless of the number of repeated measures or the degree of measurement error variance. Therefore, a sample size of 3,000 condition is excluded from further discussion of the comparison between different model specifications in the class enumeration results. Under the condition of a small distance in effect sizes (.40 vs. .70), even the condition with a sample size of 3,000 could not obtain the conventional power of .80 for successfully detecting the two latent classes using the traditional single-outcome regression mixture model. This result is consistent with the previous simulation studies, in which a one-class model was selected over the true, twoclass model in most simulations when the slope difference was small (Jaki et al., 2019). Although it is not surprising that the overall coverage rate would be relatively low in the small distance condition, the probability of selecting the two-class model increases with the number of repeated measures. Since the class detection rate was noticeably low for the small distance conditions, we further focused on the large distance condition for its accuracy in the parameter estimates.

\section{Accuracy of parameter estimates}

Tables 2 and 3 show summaries of the estimated parameters for a two-class regression mixture model for all five types of model specifications. ${ }^{8}$ Specifically, Table 2 shows the average bias in each estimated parameter (see Eq. 6), and Table 3 presents the coverage rates based on the $95 \%$ CIs constructed from each parameter's standard error. Simulations selecting the two-class model as the best-fitting model using the $\mathrm{BIC}^{9}$ are included for computing the bias as well as the coverage rate. When the sample size is 3,000, the biases for all parameter estimates are minimal across all simulation conditions. ${ }^{10}$ This is consistent with the previous research that 3,000 is an adequate number for detecting the two true classes under the present simulation condition (Jaki et al., 2019). Therefore, we compare the absolute biases for the estimated parameters across five different model specifications when the sample size is small $(n=200)$ to moderately large $(n=1,000)$. We focus on the parameters of the regression line (i.e., the

\footnotetext{
${ }^{8}$ The model results for the small distance condition can be requested from the first author.

${ }^{9}$ Given that the BIC showed more sensitive results for the class enumeration, we used the BIC to summarize the accuracy of parameter estimates.

${ }^{10} \mathrm{We}$ omitted the results for the sample size of 3,000 and the measurement error variance of $10 \%$ from Table 2 to reduce the table size for better presentation.
} 
Table 2 Bias in parameter estimates when the true two-class model was selected by the BIC under the large distance condition

\begin{tabular}{|c|c|c|c|c|c|c|c|c|c|c|c|c|c|c|}
\hline \multirow[t]{2}{*}{ Error } & \multirow[t]{2}{*}{$n$} & \multirow[t]{2}{*}{ Parameter } & \multicolumn{2}{|c|}{ Population Value } & \multicolumn{2}{|c|}{ (a) One-Time } & \multicolumn{2}{|c|}{ (b) Three-Time } & \multicolumn{2}{|c|}{ (c) Five-Time } & \multicolumn{2}{|c|}{ (d) Seven-Time } & \multicolumn{2}{|c|}{ (e) Seven-Average } \\
\hline & & & & & Class 1 & Class 2 & Class 1 & Class 2 & Class 1 & Class 2 & Class 1 & Class 2 & Class 1 & Class 2 \\
\hline \multirow[t]{9}{*}{$1 \%$} & \multirow[t]{3}{*}{200} & Intercept $\left(\beta_{0}\right)$ & .00 & .50 & -.44 & -.13 & -.24 & .03 & -.17 & .05 & -.10 & .02 & -.39 & -.02 \\
\hline & & $\mathrm{Y}$ on $\mathrm{X}\left(\beta_{1}\right)$ & .20 & .70 & -.56 & -.04 & -.22 & .02 & -.14 & .03 & -.07 & .02 & -.62 & .01 \\
\hline & & Class mean $\left(\alpha_{0}\right)$ & .00 & - & -1.38 & - & -0.44 & - & -0.24 & - & -0.16 & - & -1.20 & - \\
\hline & \multirow[t]{3}{*}{500} & Intercept $\left(\beta_{0}\right)$ & .00 & .50 & -.19 & .05 & -.11 & .01 & -.04 & .01 & -.02 & .01 & -.19 & .05 \\
\hline & & $\mathrm{Y}$ on $\mathrm{X}\left(\beta_{1}\right)$ & .20 & .70 & -.12 & .01 & -.07 & .00 & -.03 & -.01 & -.01 & .00 & -.09 & .00 \\
\hline & & Class mean $\left(\alpha_{0}\right)$ & .00 & - & -0.34 & - & -0.19 & - & -0.09 & - & -0.03 & - & -0.27 & - \\
\hline & \multirow[t]{3}{*}{1,000} & Intercept $\left(\beta_{0}\right)$ & .00 & .50 & -.09 & .01 & -.03 & .00 & -.01 & .00 & -.01 & .00 & -.09 & .01 \\
\hline & & $\mathrm{Y}$ on $\mathrm{X}\left(\beta_{1}\right)$ & .20 & .70 & -.05 & .00 & -.02 & .00 & -.01 & .00 & .00 & .00 & -.05 & .00 \\
\hline & & Class mean $\left(\alpha_{0}\right)$ & .00 & - & -0.18 & - & -0.07 & - & -0.01 & - & -0.01 & - & -0.19 & - \\
\hline \multirow[t]{9}{*}{$20 \%$} & \multirow[t]{3}{*}{200} & Intercept $\left(\beta_{0}\right)$ & .00 & .50 & -.23 & -.08 & -.14 & -.05 & -.12 & .06 & -.09 & .03 & -.27 & .03 \\
\hline & & $\mathrm{Y}$ on $\mathrm{X}\left(\beta_{1}\right)$ & .20 & .70 & -.65 & -.04 & -.21 & .02 & -.06 & .02 & -.04 & .01 & -.38 & -.03 \\
\hline & & Class mean $\left(\alpha_{0}\right)$ & .00 & - & -1.41 & - & -0.42 & - & -0.12 & - & -0.11 & - & -0.99 & - \\
\hline & \multirow[t]{3}{*}{500} & Intercept $\left(\beta_{0}\right)$ & .00 & .50 & -.17 & .01 & -.07 & .02 & -.03 & .01 & -.02 & .01 & -.14 & .01 \\
\hline & & Y on $\mathrm{X}\left(\beta_{1}\right)$ & .20 & .70 & -.15 & .01 & -.05 & .00 & -.02 & .00 & -.01 & .00 & -.10 & .00 \\
\hline & & Class mean $\left(\alpha_{0}\right)$ & .00 & - & -0.41 & - & -0.14 & - & -0.03 & - & 0.01 & - & -0.33 & - \\
\hline & \multirow[t]{3}{*}{1,000} & Intercept $\left(\beta_{0}\right)$ & .00 & .50 & -.10 & .01 & -.02 & .01 & -.01 & .00 & -.01 & .00 & -.07 & .01 \\
\hline & & $\mathrm{Y}$ on $\mathrm{X}\left(\beta_{1}\right)$ & .20 & .70 & -.05 & .00 & .00 & .00 & .00 & .00 & .00 & .00 & -.02 & .00 \\
\hline & & Class mean $\left(\alpha_{0}\right)$ & .00 & - & -0.20 & - & 0.01 & - & -0.01 & - & -0.02 & - & -0.12 & - \\
\hline \multirow[t]{9}{*}{$40 \%$} & \multirow[t]{3}{*}{200} & Intercept $\left(\beta_{0}\right)$ & .00 & .50 & -.40 & -.05 & -.19 & .01 & -.07 & .02 & -.04 & .01 & -.23 & .01 \\
\hline & & $\mathrm{Y}$ on $\mathrm{X}\left(\beta_{1}\right)$ & .20 & .70 & -.52 & -.04 & -.11 & .02 & -.09 & .01 & -.03 & .01 & -.20 & -.01 \\
\hline & & Class mean $\left(\alpha_{0}\right)$ & .00 & - & -1.39 & - & -0.34 & - & -0.20 & - & -0.03 & - & -0.67 & - \\
\hline & \multirow[t]{3}{*}{500} & Intercept $\left(\beta_{0}\right)$ & .00 & .50 & -.22 & .02 & -.07 & .02 & -.02 & .01 & -.01 & .01 & -.08 & .02 \\
\hline & & $\mathrm{Y}$ on $\mathrm{X}\left(\beta_{1}\right)$ & .20 & .70 & -.15 & .00 & -.03 & .01 & -.01 & .00 & .00 & .00 & -.03 & .01 \\
\hline & & Class mean $\left(\alpha_{0}\right)$ & .00 & - & -0.45 & - & -0.08 & - & -0.01 & - & -0.01 & - & -0.08 & - \\
\hline & \multirow[t]{3}{*}{1,000} & Intercept $\left(\beta_{0}\right)$ & .00 & .50 & -.07 & .01 & -.02 & .00 & -.01 & .00 & -.01 & .00 & -.03 & .01 \\
\hline & & Y on $\mathrm{X}\left(\beta_{1}\right)$ & .20 & .70 & -.05 & .01 & -.01 & .00 & -.01 & .00 & -.01 & .00 & -.02 & .00 \\
\hline & & Class mean $\left(\alpha_{0}\right)$ & .00 & - & -0.11 & - & -0.03 & - & -0.02 & - & -0.01 & - & -0.06 & - \\
\hline
\end{tabular}

Bias is calculated to be the difference between the average of the estimated value and the population value: $B(\overline{\hat{\beta}})=\overline{\hat{\beta}_{\text {est }}}-\beta_{\text {pop }}$. A result table including all simulation conditions can be requested from the first author.

intercept and slope) for each class because the differential effects are mainly defined by the overall pattern of regression. ${ }^{11}$ First, we describe the estimated parameters for Model A, compare the results to those for Models B through D, which used the repeated measures with the regression mixtures, and compare the results to Model E, which used the average score of seven repeated measures.

In the following section, we focus on the results for Model A, which is the traditional single-outcome regression mixture model. As is presented in Table 2, the average parameter estimates of Model A across different error variance conditions are severely biased when the sample size is equal to or less than 1,000. Although the intercept coefficient and the regression weight for

\footnotetext{
${ }^{11}$ The average estimates for factor variance and residual variance for all conditions can also be obtained from the first author on request.
}

the large effect class (Class 2) are relatively well estimated across all conditions, except for the sample size of 200, those for the small effect class (Class 1) are substantially biased, depending on the model specification. When the population parameters for intercept and slope are $\beta_{00}=.00$ and $\beta_{10}=.20$ for Class 1 and $\beta_{01}=.50$ and $\beta_{11}=.70$ for Class 2 , the average parameter biases across all sample size and measurement variance conditions, $B$, for the two estimates are $B\left(\beta_{00}\right)=-.20$ and $B\left(\beta_{10}\right)=-.23$ for Class 1 , and $B\left(\beta_{01}\right)=-.01$ and $B\left(\beta_{11}\right)=-.01$ for Class 2, respectively. That is, parameter estimates for Class 1 are substantially downward-biased, whereas those for Class 2 are relatively stable. The class mean, which is the log of the odds of being assigned to Class 1 , is also severely biased to be $B\left(\alpha_{0}\right)=-.65$ (population class mean $=.00$ ). That is, about two times more subjects (66\%) are assigned to be in Class 2, which thus dominates the class enumeration results. 
Table 3 Coverage rates ${ }^{\mathrm{a}}$ of the parameter estimates when the true two-class model was selected by the BIC under the large distance condition

\begin{tabular}{|c|c|c|c|c|c|c|c|c|c|c|c|c|c|c|}
\hline \multirow[t]{2}{*}{ Error } & \multirow[t]{2}{*}{$n$} & \multirow[t]{2}{*}{ Parameter } & \multicolumn{2}{|c|}{ Population Value } & \multicolumn{2}{|c|}{ (a) One-Time } & \multicolumn{2}{|c|}{ (b) Three-Time } & \multicolumn{2}{|c|}{ (c) Five-Time } & \multicolumn{2}{|c|}{ (d) Seven-Time } & \multicolumn{2}{|c|}{ (e) Seven-Average } \\
\hline & & & & & Class 1 & Class 2 & Class 1 & Class 2 & Class 1 & Class 2 & Class 1 & Class 2 & Class 1 & Class 2 \\
\hline \multirow[t]{9}{*}{$1 \%$} & 200 & Intercept $\left(\beta_{0}\right)$ & .00 & .50 & .37 & .56 & .67 & .79 & .76 & .81 & .88 & .88 & .46 & .51 \\
\hline & & $\mathrm{Y}$ on $\mathrm{X}\left(\beta_{1}\right)$ & .20 & .70 & .42 & .42 & .74 & .71 & .86 & .86 & .92 & .88 & .51 & .49 \\
\hline & & Class mean $\left(\alpha_{0}\right)$ & .00 & - & 0.39 & - & 0.67 & - & 0.72 & - & 0.82 & - & 0.29 & \\
\hline & 500 & Intercept $\left(\beta_{0}\right)$ & .00 & .50 & .85 & .84 & .89 & .90 & .94 & .92 & .95 & .95 & .82 & .86 \\
\hline & & $\mathrm{Y}$ on $\mathrm{X}\left(\beta_{1}\right)$ & .20 & .70 & .81 & .82 & .90 & .87 & .93 & .92 & .95 & .94 & .81 & .77 \\
\hline & & Class mean $\left(\alpha_{0}\right)$ & .00 & - & 0.76 & - & 0.84 & - & 0.91 & - & 0.94 & - & 0.74 & \\
\hline & 1,000 & Intercept $\left(\beta_{0}\right)$ & .00 & .50 & .90 & .93 & .94 & .94 & .94 & .95 & .96 & .96 & .89 & .94 \\
\hline & & $\mathrm{Y}$ on $\mathrm{X}\left(\beta_{1}\right)$ & .20 & .70 & .93 & .88 & .93 & .93 & .93 & .96 & .93 & .96 & .94 & .89 \\
\hline & & Class mean $\left(\alpha_{0}\right)$ & .00 & - & 0.88 & - & 0.94 & - & 0.94 & - & 0.95 & - & 0.90 & \\
\hline \multirow[t]{9}{*}{$20 \%$} & 200 & Intercept $\left(\beta_{0}\right)$ & .00 & .50 & .36 & .53 & .62 & .67 & .86 & .83 & .89 & .88 & .57 & .61 \\
\hline & & $\mathrm{Y}$ on $\mathrm{X}\left(\beta_{1}\right)$ & .20 & .70 & .36 & .42 & .60 & .67 & .89 & .87 & .90 & .88 & .65 & .67 \\
\hline & & Class mean $\left(\alpha_{0}\right)$ & .00 & - & 0.40 & - & 0.60 & - & 0.82 & - & 0.87 & - & 0.57 & \\
\hline & 500 & Intercept $\left(\beta_{0}\right)$ & .00 & .50 & .82 & .84 & .92 & .94 & .95 & .94 & .94 & .94 & .87 & .89 \\
\hline & & Y on $\mathrm{X}\left(\beta_{1}\right)$ & .20 & .70 & .86 & .76 & .92 & .88 & .94 & .93 & .93 & .94 & .90 & .83 \\
\hline & & Class mean $\left(\alpha_{0}\right)$ & .00 & - & 0.81 & - & 0.93 & - & 0.95 & - & 0.95 & - & 0.85 & \\
\hline & 1,000 & Intercept $\left(\beta_{0}\right)$ & .00 & .50 & .91 & .91 & .94 & .95 & .95 & .95 & .94 & .97 & .91 & .94 \\
\hline & & $\mathrm{Y}$ on $\mathrm{X}\left(\beta_{1}\right)$ & .20 & .70 & .89 & .89 & .92 & .94 & .93 & .95 & .96 & .97 & .92 & .89 \\
\hline & & Class mean $\left(\alpha_{0}\right)$ & .00 & - & 0.84 & - & 0.93 & - & 0.93 & - & 0.94 & - & 0.88 & \\
\hline \multirow[t]{9}{*}{$40 \%$} & 200 & Intercept $\left(\beta_{0}\right)$ & .00 & .50 & .37 & .46 & .74 & .82 & .84 & .81 & .89 & .89 & .61 & .70 \\
\hline & & Y on $\mathrm{X}\left(\beta_{1}\right)$ & .20 & .70 & .43 & .37 & .79 & .78 & .82 & .81 & .87 & .86 & .65 & .68 \\
\hline & & Class mean $\left(\alpha_{0}\right)$ & .00 & - & 0.37 & - & 0.67 & - & 0.77 & - & 0.83 & - & 0.62 & \\
\hline & 500 & Intercept $\left(\beta_{0}\right)$ & .00 & .50 & .78 & .78 & .92 & .93 & .96 & .93 & .96 & .94 & .87 & .87 \\
\hline & & $\mathrm{Y}$ on $\mathrm{X}\left(\beta_{1}\right)$ & .20 & .70 & .80 & .77 & .89 & .91 & .94 & .94 & .95 & .95 & .91 & .83 \\
\hline & & Class mean $\left(\alpha_{0}\right)$ & .00 & - & 0.71 & - & 0.91 & - & 0.96 & - & 0.96 & - & 0.85 & \\
\hline & 1,000 & Intercept $\left(\beta_{0}\right)$ & .00 & .50 & .90 & .89 & .95 & .93 & .96 & .93 & .96 & .94 & .94 & .94 \\
\hline & & Y on $\mathrm{X}\left(\beta_{1}\right)$ & .20 & .70 & .94 & .90 & .94 & .93 & .96 & .95 & .95 & .95 & .94 & .92 \\
\hline & & Class mean $\left(\alpha_{0}\right)$ & .00 & - & 0.89 & - & 0.95 & - & 0.95 & - & 0.94 & - & 0.94 & \\
\hline
\end{tabular}

${ }^{\text {a }}$ Coverage rate is calculated based on the $95 \%$ confidence interval for each simulation dataset with the corresponding $S E$

The coverage rates also support the findings for the bias in parameter estimates. Table 3 shows that the coverage rates for the population parameters under the small sample size condition are very low (ranging between .36 and .56 ), meaning that the chances of recovering the true values are low for the sample size of 200, even when the two-class model is selected as the best-fitting model. On the other hand, where the major biases in the parameter estimates are driven by Class 1 (see Table 2), the coverage rate is relatively consistent between the two classes. The average coverage rate of the $95 \%$ CIs for intercepts and slopes for Class 1 are .72 and .74, while they are .77 and .71 for Class 2, respectively. The reason is that the standard errors for the estimated parameters for Class 1 are much larger than those for Class 2, given the former's instability and smaller class size.

Overall, the results show that small sample size $(n=200)$ is a serious problem in regular regression mixture models because it produces biased parameter estimates, such as switched directions for the regression weights (a positive effect showing as negative) and biased class proportions. Across all conditions of measurement error variance, the parameter estimates for Class 1 are downward-biased (ranging between - .65 and.23 ), and the power to detect the population parameter is low (ranging between .36 and .43).

Adding additional time points When adding additional time points, accuracy of the parameter estimates is substantially improved on average across all error variance conditions under the small to moderately large sample size conditions. The average biases for the intercept and slope for Class 1 are reduced to $B\left(\beta_{00}\right)=-.09$ and $B\left(\beta_{10}\right)=-.07$ when adding two more time points (Model B). In the same pattern, when increasing the number of repeated measures to five or seven, the absolute biases for the intercept and slope for Class 1 steadily decrease, to $B\left(\beta_{00}\right)=-.05$ and $B\left(\beta_{10}\right)=-.04$ for Model $C$ and $B\left(\beta_{00}\right)=-.03$ and $B\left(\beta_{10}\right)=-.02$ for Model D, respectively. 
Conversely, the coverage rates of the $95 \%$ CIs to detect the population value increase as the number of repeated measures increases. Across all sample size and measurement variance conditions, the average coverage rates of all parameter estimates $\left(\beta_{00}, \beta_{10}, \beta_{01}, \beta_{11}\right.$, and $\left.\alpha_{0}\right)$ are increased gradually by adding more repeated measures into the regression mixture analyses. For example, when a week of data (seven repeated measures) are used in regression mixture models instead of a single time point measure, biases in the parameter estimates are dramatically reduced, to about seven times smaller for the intercept $(-.20$ to -.03$)$ and 11 times smaller for the regression weight $(-.23$ to -.02$)$, on average, while the coverage rate is increased to .95 .

More specifically, under the condition of $20 \%$ measurement error variance with a sample size of 500, for example, the average estimates of the intercept and slope for Class 1 for Model A are $\beta_{00}=-.17\left(M_{S E}=.17\right)$ and $\beta_{10}=.06\left(M_{S E}\right.$ $=.14)$, which are seriously underestimated. That is, the statistical significance of a small effect (population value of $\beta_{10}=.20$ ) cannot even be detected in most simulation conditions, but instead is regarded as "no effect." Notably, the standard deviation of the estimated regression weights for Class 1 across simulations is .30 , which is substantial, indicating that the regression coefficient for the small effect class is unstable and is estimated to be negative in many simulations. As compared to Model A, when using the seven repeated measures in regression mixtures (Model D), the average regression weight for Class 1 under the same conditions $(n=500$, error variance $=20 \%)$ is $\beta_{10}=$ $.19\left(M_{S E}=.09\right)$, which is very close to the population value. In terms of statistical significance, this clearly shows the improved accuracy for estimating the regression weight for the smaller effect group with the average standard error of .09 when using the repeated measures approach. The standard deviation of the regression weights is .09 , as well, which is greatly reduced from that of Model A $(S D=.30)$, showing the improved model stability.

Likewise, the computed bias in class means is greatly reduced by increasing the number of repeated measures in the regression mixture models. When adding more repeated measures into the model, the average bias in class means, $B\left(\alpha_{0}\right)$, is reduced to $-.19,-.08$, and -.04 for Models B, C, and D, respectively, meaning that more subjects are correctly assigned to be in Class 1, given the increased number of repeated measures. This finding is also supported by the increased coverage rates shown in Table 3.

Using the average value of repeated measures Next, we examine whether using an average score of repeated measures has an advantage over a single time measure in the accuracy of parameter estimates. As is shown in Tables 2 and 3, Model E (using the average score of the seven repeated measures model) shows no clear advantage over Model A. Although the size of the biases for the intercept and slope for Class $1\left[B\left(\beta_{00}\right)=-\right.$ .16 and $\left.B\left(\beta_{10}\right)=-.16\right]$ are smaller than those from Model $\mathrm{A}$ $\left[B\left(\beta_{00}\right)=-.20\right.$ and $\left.B\left(\beta_{10}\right)=-.23\right]$, the estimated parameters are still seriously biased and underestimated, as compared to Models B, C, and D. The absolute biases in the class mean $\left[B\left(\alpha_{0}\right)=-.43\right]$ and the coverage rate (.74) for Model $\mathrm{E}$ also show that the benefit of using the average score of the repeated measures is limited as compared to the repeated measures regression mixture approach.

\section{Application: Use of a repeated measures regression mixture model to Sleep Research Project data}

To demonstrate the use of the repeated measures regression mixture model, we utilized data from the University of Memphis Sleep Research Project epidemiological survey of sleep and daytime functioning (Lichstein, Durrence, Riedel, Taylor, \& Bush, 2004). Although the overall association between morbidity and sleep disturbances has been studied as a means of calibrating health risk (Altevogt \& Colten, 2006; Nilsson, Rööst, Engström, Hedblad, \& Berglund, 2004; Taylor et al., 2007), the literature helping us understand heterogeneity in the effects of daytime functioning on sleep quality, or vice versa, has been limited. It is important to understand these potential differential effects, because daytime functioning (e.g., depression, anxiety, and fatigue) affects the perceived sleep impairment, which has been identified as a significant factor in insomnia treatment-seeking behavior and increased use of healthcare (Moul et al., 2002; Ustinov et al., 2010).

In the present application study, regression mixture models were used to explore and better understand the potential differential effects of daytime functioning on sleep quality. Specifically, we illustrate the use of repeated measures regression mixtures to identify qualitatively different groups of people who differ in the effect of cognitive and behavioral symptoms of depression on sleep efficiency. A total of 771 participants, ranging in age from 20 to 98 years, participated in the study and were included in the data analysis. More details on the demographic and socio-economic characteristics of participants are available from Lichstein et al. (2004). Two weeks (14 days) of sleep diary were measured using a questionnaire. Among nine sleep measures (e.g., the time in minutes to fall asleep, the number of awakenings during the night, total sleep time, total sleep in bed, etc.), we employed the sleep efficiency percentages for the present demonstration, which represent the ratio of total sleep time to total time in bed $\times 100$, indicating overall sleep quality. Depression severity was measured using the Beck Depression Inventory (BDI), consisting of 21 items (Taylor et al., 2007).

To demonstrate the use of repeated measures regression mixture models in the present application, we used the eight days of weekday data (Monday through Thursday for two 


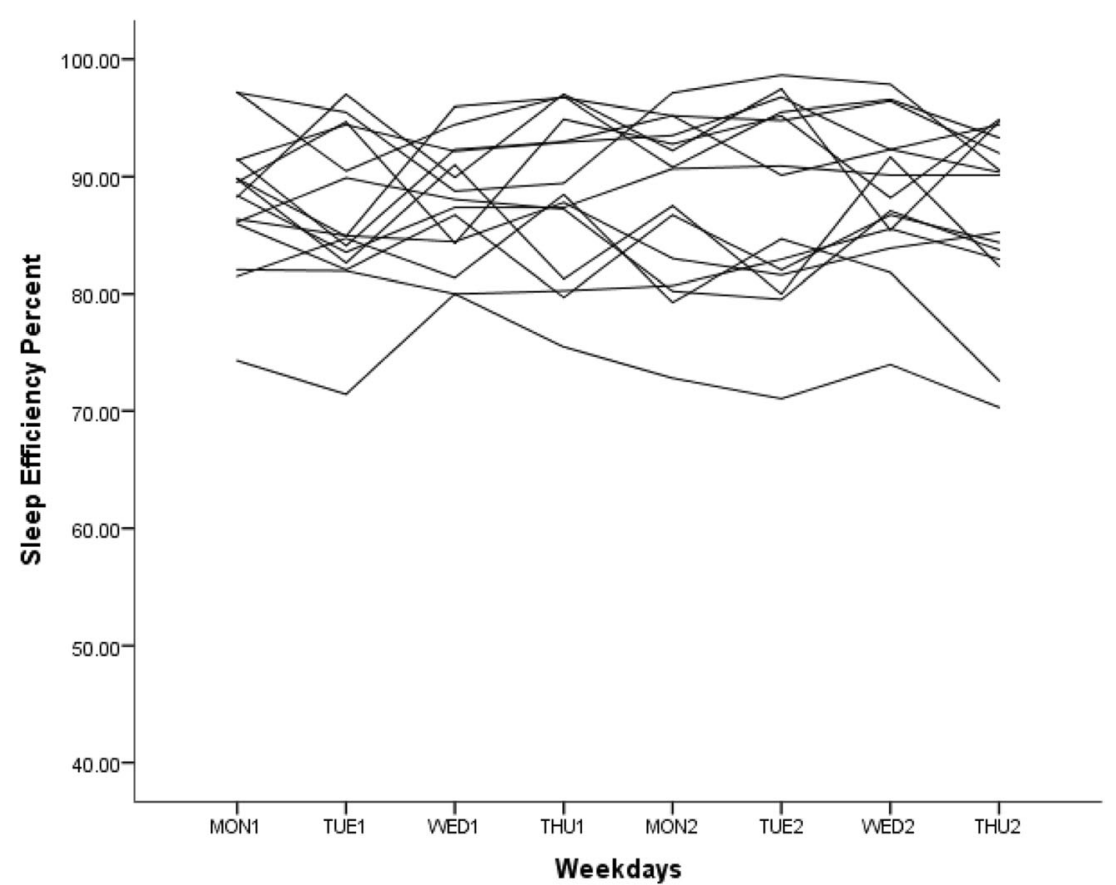

Fig. 3 Spaghetti plot for the two weeks of weekday data on the sleep efficiency scale $(n=15)$

weeks), to focus on the differential effect of daytime functioning on sleep quality during weekdays. Since previous research has shown some different sleep patterns between weekdays and weekends (Adam, Snell, \& Pendry, 2007; Vitale et al., 2014), we excluded the weekend data for the present illustration, to remove the potential complications from combining weekday and weekend data in the same analysis. First, to examine whether there is any systematic change in sleep efficiency over time during weekdays, we plotted the individual trajectories across eight days in data from a randomly selected 15 subjects. Figure 3 shows no particular pattern in the repeated measures of sleep quality over time, other than random fluctuations. Next, we analyzed an intercept-only model under the MLM framework, to assess the intraclass correlation coefficient (ICC), which is a gauge of the correlation among the observations within subjects (Raudenbush \& Bryk, 2002). The calculated ICC was $.49,{ }^{12}$ indicating that about half of the variance was found at the between-subjects level. We were specifically interested in understanding the between-subjects variance, to the extent that we hypothesized that the betweensubjects variance would be differentially explained by daytime functioning (i.e., depression), on the basis of the unobserved latent classes. Figure 4 shows the model specification for the repeated measures regression mixtures for sleep efficiency outcome, with depression as the predictor. As is shown in Fig. 4, each sleep efficiency measure was treated as an indicator of a latent intercept factor of sleep efficiency, with

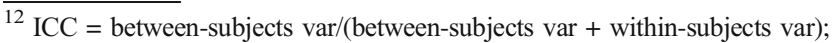
that is, $.49=79.36 /(79.36+81.10)$, from the results. a fixed factor loading of 1; thus, the latent variable represents the average sleep efficiency across eight repeated measures. The within-subjects residual variances $\left(\sigma^{2}{ }_{k}\right)$ were constrained to be equal across time points but were allowed to differ across classes, and no covariances were estimated (i.e., ID structure $\left.{ }^{13}\right)$. The intercept $\left(\beta_{0 k}\right)$, slope $\left(\beta_{1 k}\right)$, and between-subjects residual variance $\left(\psi_{k}\right)$ were estimated to be class-specific. A traditional regression analysis (one-class) was conducted, and the results were compared to a series of regression mixture models (two-class, three-class, four-class, and five-class). Mplus 7.3 (L. K. Muthén \& Muthén, 1998-2012) was used for all data analyses, and the BIC and ABIC were used for model comparisons. ${ }^{14}$

Table 4 presents the results of the repeated measures regression mixture model investigating the differential effect of depression on sleep impairment. On the basis of the BICs and ABICs for all four models, the four-class model fit the data best, with criterion values of 42,161 and 42,100 , respectively. ${ }^{15}$ However, the Mplus results inform us that the solution may not be trustworthy, given that the best log-likelihood value was not replicated, which could be a sign of a local solution. Thus, we increased the random start value to be 500 (the Mplus default value is 20), which was suggested by the program. The result still warned that there was a

\footnotetext{
$\overline{13}$ To reduce the complexity of the model specifications, the simplest ID error variance structure was adopted in the current study for demonstration purpose. ${ }^{14}$ The Mplus syntax for analyzing the present models is provided in the Appendix, for applied researchers.

${ }^{15}$ We also analyzed the five-class model, but the results did not converge, which can be a sign that we are trying to extract too many classes.
} 


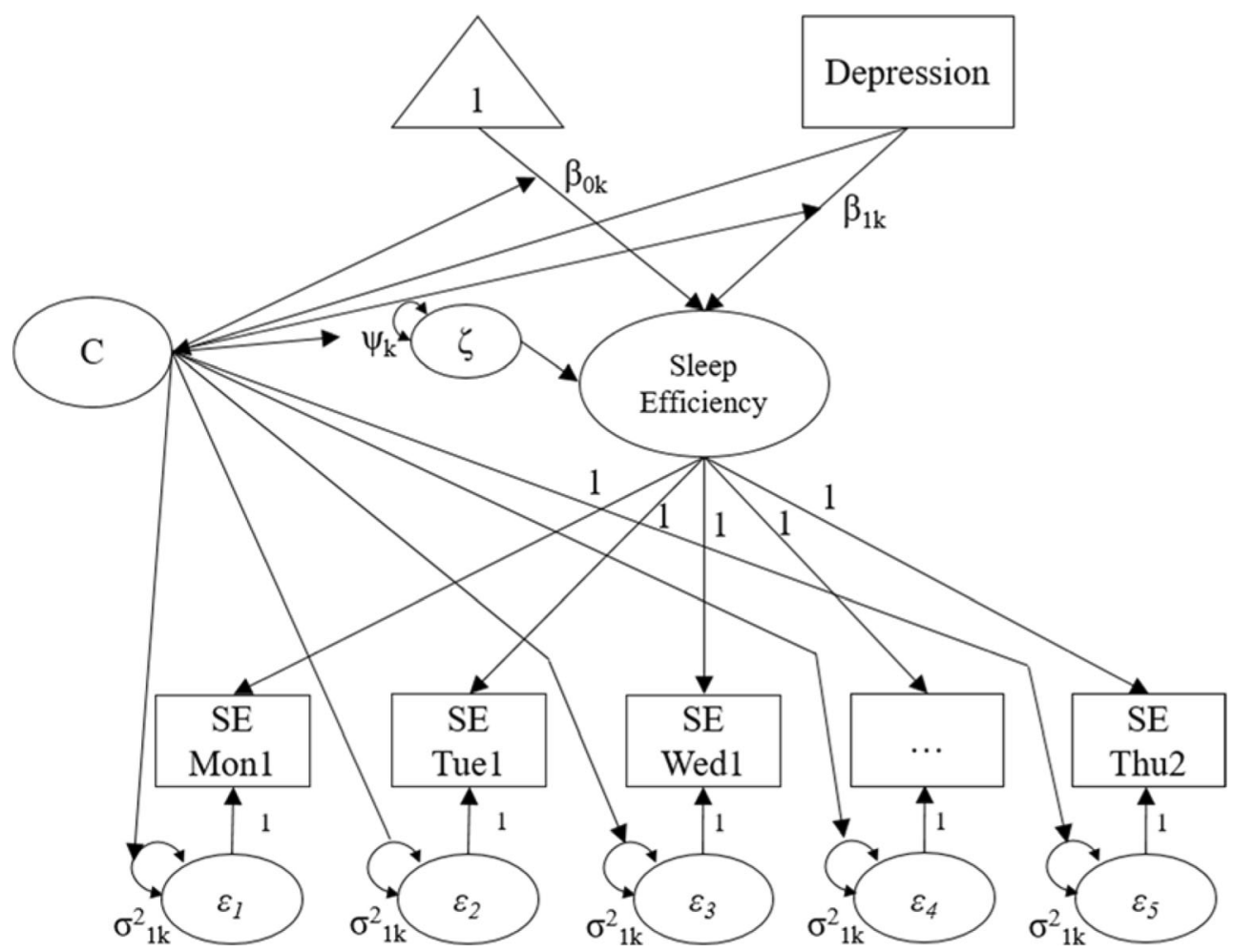

Fig. 4 Repeated measures regression mixture model for the sleep efficiency measure, with depression as the predictor

nonconvergence issue. Not surprisingly, the five-class model did not converge, which often is a sign that too many classes are being requested to be extracted. Thus, we present the results from both the three- and four-class models for readers of this article (see Table 4), instead of presenting only one result over the other. Our interpretation of the model results is based on the three-class model, which converged with no issue.
As is shown in Table 4, about half of the participants were classified as being in Class 1, named as the normal sleeper group, with a $90 \%$ sleep efficiency level, on average, and a small negative effect of depression. About $23 \%$ of the participants were classified as being in Class 2, named as the healthy sleeper group, with the highest sleep efficiency level, on average (95.4\%), and a smaller effect of depression on their sleep

Table 4 Parameter estimates from three-class model for Sleep Research Project data

\begin{tabular}{|c|c|c|c|c|c|c|c|c|}
\hline \multirow{3}{*}{$\begin{array}{l}\text { Number of latent classes } \\
\text { BIC } \\
\text { ABIC }\end{array}$} & \multirow{3}{*}{$\begin{array}{l}\text { One-Class } \\
48,955 \\
48,946\end{array}$} & \multirow[t]{2}{*}{ Two-Class } & \multicolumn{2}{|c|}{ Three-Class } & \multicolumn{2}{|c|}{ Four-Class } & \multicolumn{2}{|c|}{ Five-Class } \\
\hline & & & 43,074 & 42,346 & 42,161 & & Non-co & ged \\
\hline & & & 43,045 & 42,301 & 42,100 & & & \\
\hline Three-class model solution & \multicolumn{2}{|c|}{ Class 1 Normal Sleeper } & \multicolumn{2}{|c|}{ Class 2 Healthy Sleeper } & \multicolumn{2}{|c|}{ Class 3 Sleep Impaired } & & \\
\hline Class proportion & \multicolumn{2}{|c|}{$48.64 \%$} & \multicolumn{2}{|c|}{$22.96 \%$} & \multicolumn{2}{|c|}{$28.41 \%$} & & \\
\hline Parameter Estimates & Coeff. & $S E$ & Coeff. & $S E$ & Coeff. & $S E$ & & \\
\hline Intercept & 89.37 & 0.47 & 95.40 & 0.34 & 79.06 & 1.08 & & \\
\hline Depression & -0.16 & 0.06 & -0.13 & 0.04 & -0.35 & 0.09 & & \\
\hline Factor variance & 14.58 & 1.87 & 4.61 & 0.82 & 56.58 & 9.59 & & \\
\hline Residual variance & 35.71 & 3.52 & 4.58 & 0.60 & 218.63 & 18.43 & & \\
\hline Four-class model solution & \multicolumn{2}{|l|}{ Class 1} & \multicolumn{2}{|l|}{ Class 2} & \multicolumn{2}{|l|}{ Class 3} & \multicolumn{2}{|c|}{ Class 4} \\
\hline Class proportion & \multicolumn{2}{|l|}{$35.28 \%$} & \multicolumn{2}{|l|}{$14.66 \%$} & \multicolumn{2}{|l|}{$18.16 \%$} & \multicolumn{2}{|c|}{$31.91 \%$} \\
\hline Parameter Estimates & Coeff. & $S E$ & Coeff. & $S E$ & Coeff. & $S E$ & Coeff. & $S E$ \\
\hline Intercept & 86.71 & 0.73 & 96.19 & 0.34 & 76.37 & 1.51 & 91.87 & 0.56 \\
\hline Depression & -0.22 & 0.05 & -0.11 & 0.05 & -0.40 & 0.12 & -0.10 & 0.03 \\
\hline Factor variance & 14.73 & 3.04 & 2.57 & 0.59 & 49.59 & 11.47 & 7.00 & 1.11 \\
\hline Residual variance & 67.06 & 7.64 & 2.85 & 0.67 & 275.36 & 26.33 & 16.49 & 2.65 \\
\hline
\end{tabular}


quality. In other words, the participants in Class 2 were not greatly affected by the severity of depression in their sleep efficiency, while overall they had a good sleep quality. About $28.4 \%$ of the participants were classified as being in Class 3, named as a sleep-impaired group, with the lowest level of sleep efficiency (79\%) and the largest negative effect of depression on the quality of their sleep. The people in Class 3 were mostly the focus group for further investigation in health and clinical research, to find out who they were and why they were vulnerable to daytime functioning and psychological traits on their sleep. As is shown in the present demonstration, regression mixture models allow applied researchers to investigate potential differential effects of interest in repeated measures outcomes.

\section{Discussion}

Given the complexity of human nature, people respond differently to the same context. Regression mixture models are a powerful tool for identifying the unobserved class of subjects based on the differential effects of interest without a priori hypothesis. This study extends the use of traditional regression mixture models with a single observed outcome to a latent construct of repeated measures outcomes. The present approach for repeated measures regression mixture model can be considered as a special type of factor mixture modeling (Lubke \& Muthén, 2005) in which a population's heterogeneity is studied through the effect of predictor $X$ on the latent construct $\eta$, having the indicator $Y$ as the repeated measures. Where factor mixture modeling is a broader latent class approach, which covers almost every type of population heterogeneity, repeated measures regression mixture models are specialized to detect the effect heterogeneity, which focuses more on the substantive questions.

Although regression mixture models are a powerful tool for identifying the latent class showing the differential effects of interest, with only a single outcome, the required sample size is very large, and is impractical in many applications (Jaki et al., 2019; Van Horn et al., 2015). The present approach using repeated measures suggests an alternative solution for improving the power to search for heterogeneous groups defined by the effect of interest. We note that this approach is especially recommended for those who are interested in the effect of heterogeneity with a small sample size when they have or are able to collect additional measurements from the same subjects. Collecting repeated measures data from the same subjects can be as difficult as increasing the sample size in many longitudinal studies, especially with measurements over longer period of time (e.g., attrition, relocation, mortality, etc.). The present method is particularly interested in using the repeated measures with relatively short time-interval, in which the main purpose of collecting the repeated measures is on achieving more stable measurements for the subjects rather than examining the change over time (e.g., daily diary of patients' pain severity, sleep diary, diary of physical activity, etc.). As is shown in the results, introducing repeated measures into regression mixture models greatly improves the likelihood of identifying the correct number of latent classes, reduces bias in parameter estimates, and improves efficiency and stability of the results of analysis.

Whereas prior recommendations suggested that regression mixture models require samples of well over 1,000 participants, even under the condition of a large distance in effect sizes between the classes (Van Horn et al., 2015), the present repeated measures regression mixture models allow for samples as low as 200 participants when the classes have a separation like that simulated herein. Specifically, the results show that at the lowest sample size considered, 200, more repeated measures are needed for problems like those in our simulation model; notable bias remained in the class with a weaker regression effect, even with five (but not seven) repeated measures. At samples closer to 500, the results were reasonably stable and bias-free when a large distance between two classes was conditioned. Thus, the use of repeated measures regression mixture modeling greatly extends the sample sizes for which regression mixtures are feasible, if repeated measures are available. However, it is important to note that the results are dependent on including the repeated measures for specifying the regression mixture model, rather than using the average score as a single outcome. The results show that the use of average scores for the repeated measures in the regression mixture produces only small, and not substantial, gains in class enumeration accuracy or bias of parameter estimates. Another important point is that adding more repeated measures into the regression mixture models has a greater impact when the measures are relatively unstable. In other words, if the measures are highly stable across time, such as repeated measures of body mass index, the benefit of having the additional time point is limited given the small amount of extra information from the multiple time points.

\section{Limitations}

As in most simulation studies, there are some limitations regarding the simulation conditions in the present study. First, we used two classes for the population model in the present study. Because the study conditions were already complex, with five different model specifications for each simulated dataset, we simplified the simulation conditions for generating the true population model. Although we looked at a relatively small set of the possible regression mixtures, we believe that the major findings of the present study (i.e., improved power and accuracy for enumerating classes and estimating parameters in regression mixtures when using the repeated measures) would also hold for extended conditions. Second, we 
elucidated this model using a relatively simple example in which there was no time trend. This, of course, can easily be extended to the case in which there was a time trend in the outcome. In this case, the repeated measures mixture is defined on the intercept term and/or the slope coefficient of the growth curve. The results of the present simulations should generalize to this slight extension, as long as the growth trend is modeled as a nuisance and constrained to be equal across classes. When the researcher's question concerns heterogeneity of the growth trajectory, however, growth mixture models can be further considered for analyzing the data. We note that the substantive question is largely shifted from the differential effects to the differential trajectories, in this case. More research will be needed to understand the case in which a growth mixture approach is instead more appropriate.

We purposefully constrained the total variance to be 1 for both latent classes across all simulation conditions, to maintain a constant effect size, regardless of the different conditions in measurement error variance. By doing this, the increase in measurement error variance, which can possibly have a negative impact on the regression mixtures, was confounded with the reduction in the intercept variance of the repeated measures, which could possibly have a positive impact on the results by providing more information for estimation from the multiple outcomes. Also, we used the simplest identity variance-covariance matrix for the residual error variance structure. It can be extended to more complex error variance structures in future research, to examine whether the present approach still captures the latent classes appropriately and performs successfully under more complex scenarios.

For the application study, we simplified the model to be an unconditional regression mixture with only the main predictor, depression, without any other covariates, given the purpose of demonstration. We note that the results are intended to demonstrate the use of repeated measures regression mixtures rather than to thoroughly examine heterogeneity in the effects of depression on sleep efficiency. Thus, findings of the present application study using the empirical data (i.e., three different types of sleepers, given the effect of depression) should be interpreted by means of a more thorough investigation.

\section{Conclusion}

Regression mixtures have been shown to be effective at finding differential effects in behavioral and health outcomes; however, large sample sizes are necessary in order to have stable and robust findings (Van Horn et al., 2015). Our repeated measures regression mixture approach holds extreme promise of reducing the size of the sample of individuals needed in order to use this methodology, as long as repeated measures are available. With the increase in repeated measures data that is associated with daily diary, logging, and ecological momentary data, there will be increasing opportunities to use this approach.

Author note None of the data or materials for the experiments reported here are available, and the experiment was not preregistered.

\section{References}

Adam, E. K., Snell, E. K., \& Pendry, P. (2007). Sleep timing and quantity in ecological and family context: A nationally representative timediary study. Journal of Family Psychology, 21, 4-19. https://doi.org/ 10.1037/0893-3200.21.1.4

Altevogt, B. M., \& Colten, H. R. (2006). Sleep disorders and sleep deprivation: An unmet public health problem. Washington, DC: National Academies Press.

Bauer, D. J., \& Curran, P. J. (2003). Distributional assumptions of growth mixture models: Implications for overextraction of latent trajectory classes. Psychological Methods, 8, 338-363. https://doi.org/10. 1037/1082-989X.8.3.338

Duncan, T. E., Duncan, S. C., \& Strycker, L. A. (2013). An introduction to latent variable growth curve modeling: Concepts, issues, and application, Basingstoke: Routledge.

Dyer, W. J., Pleck, J., \& McBride, B. (2012). Using mixture regression to identify varying effects: A demonstration with parental incarceration. Journal of Marriage and Family, 74, 1129-1148.

Grimm, K. J., Ram, N., \& Estabrook, R. (2017). Growth modeling: Structural equation and multilevel modeling approaches. New York: Guilford Press.

Jaki, T., Kim, M., Lamont, A. E., George, M., Chang, C., Feaster, D. J., \& Van Horn, M. L. (2019). The effects of sample size on the estimation of regression mixture models. Educational and Psychological Measurement, 79, 358-384. https://doi.org/10.1177/ 0013164418791673

Jung, T., \& Wickrama, K. (2008). An introduction to latent class growth analysis and growth mixture modeling. Social and Personality Psychology Compass, 2, 302-317.

Kliegel, M., \& Zimprich, D. (2005). Predictors of cognitive complaints in older adults: A mixture regression approach. European Journal of Ageing, 2, 13-23.

Kohli, N., Harring, J. R., \& Zopluoglu, C. (2016). A finite mixture of nonlinear random coefficient models for continuous repeated measures data. Psychometrika, 81, 851-880.

Lamont, A. E., Vermunt, J. K., \& Van Horn, M. L. (2016). Regression mixture models: Does modeling the covariance between independent variables and latent classes improve the results? Multivariate Behavioral Research, 51, 35-52.

Lanza, S. T., Cooper, B. R., \& Bray, B. C. (2014). Population heterogeneity in the salience of multiple risk factors for adolescent delinquency. Journal of Adolescent Health, 54, 319-325. https://doi. org/10.1016/j.jadohealth.2013.09.007

Lanza, S. T., Kugler, K. C., \& Mathur, C. (2011). Differential effects for sexual risk behavior: An application of finite mixture regression. Open Family Studies Journal, 4, 81-88.

Lee, E. J. (2013). Differential susceptibility to the effects of child temperament on maternal warmth and responsiveness. Journal of Genetic Psychology: Research and Theory on Human Development, 174, 429-449.

Lichstein, K. L., Durrence, H. H., Riedel, B. W., Taylor, D. J., \& Bush, A. J. (2004). Epidemiology of sleep: Age, gender, and ethnicity. Mahwah: Erlbaum. 
Lubke, G. H., \& Muthén, B. (2005). Investigating population heterogeneity with factor mixture models. Psychological Methods, 10, 21 39. https://doi.org/10.1037/1082-989X.10.1.21

McDonald, S. E., Shin, S., Corona, R., Maternick, A., Graham-Bermann, S. A., Ascione, F. R., \& Williams, J. H. (2016). Children exposed to intimate partner violence: Identifying differential effects of family environment on children's trauma and psychopathology symptoms through regression mixture models. Child Abuse \& Neglect, 58, $1-$ 11.

McLachlan, G., \& Peel, D. (2000). Finite mixture models. New York: Wiley.

Moul, D. E., Nofzinger, E. A., Pilkonis, P. A., Houck, P. R., Miewald, J. M., \& Buysse, D. J. (2002). Symptom reports in severe chronic insomnia. Sleep, 25, 548-558.

Muthén, B. (2004). Latent variable analysis: Growth mixture modeling and related techniques for longitudinal data. In D. Kaplan (Ed.), The Sage handbook of quantitative methodology for the social sciences (pp. 345-368). Thousand Oaks: Sage.

Muthén, B., \& Muthén, L. K. (2000). Integrating person-centered and variable-centered analyses: Growth mixture modeling with latent trajectory classes. Alcoholism: Clinical and Experimental Research, 24, 882-891.

Muthén, L. K., \& Muthén, B. O. (1998-2012). Mplus user's guide (7th). Los Angeles: Muthén \& Muthén.

Ng, S. K., McLachlan, G. J., Wang, K., Ben-Tovim Jones, L., \& Ng, S. W. (2006). A mixture model with random-effects components for clustering correlated gene-expression profiles. Bioinformatics, 22, 1745-1752. https://doi.org/10.1093/bioinformatics/btl165

Nilsson, P. M., Rööst, M., Engström, G., Hedblad, B., \& Berglund, G. (2004). Incidence of diabetes in middle-aged men is related to sleep disturbances. Diabetes Care, 27, 2464-2469.

Nylund, K. L., Asparouhov, T., \& Muthén, B. O. (2007). Deciding on the number of classes in latent class analysis and growth mixture modeling: A Monte Carlo simulation study. Structural Equation Modeling, 14, 535-569.

R Core Team. (2017). R: A language and environment for statistical computing. Vienna, Austria: R Foundation for Statistical Computing. Retrieved from https://www.R-project.org

Raudenbush, S. W., \& Bryk, A. S. (2002). Hierarchical linear models: Applications and data analysis methods (2nd). Thousand Oaks: Sage.

Schmiege, S. J., \& Bryan, A. D. (2016). Heterogeneity in the relationship of substance use to risky sexual behavior among justice-involved youth: A regression mixture modeling approach. Aids and Behavior, $20,821-832$

Schwarz, G. (1978). Estimating the dimension of a model. Annals of Statistics, 6, 461-464.

Sclove, S. L. (1987). Application of model-selection criteria to some problems in multivariate analysis. Psychometrika, 52, 333-343.
Silinskas, G., Kiuru, N., Tolvanen, A., Niemi, P., Lerkkanen, M.-K., \& Nurmi, J.-E. (2013). Maternal teaching of reading and children's reading skills in Grade 1: Patterns and predictors of positive and negative associations. Learning and Individual Differences, 27, 54-66. https://doi.org/10.1016/j.lindif.2013.06.011

Silinskas, G., Pakarinen, E., Niemi, P., Lerkkanen, M.-K., Poikkeus, A.M., \& Nurmi, J.-E. (2016). The effectiveness of increased support in reading and its relationship to teachers' affect and children's motivation. Learning and Individual Differences, 45, 53-64.

Sperrin, M., Jaki, T., \& Wit, E. (2010). Probabilistic relabelling strategies for the label switching problem in Bayesian mixture models. Statistics in Computing, 20, 357-366. https://doi.org/10.1007/ s11222-009-9129-8

Taylor, D. J., Mallory, L. J., Lichstein, K. L., Durrence, H. H., Riedel, B. W., \& Bush, A. J. (2007). Comorbidity of chronic insomnia with medical problems. Sleep, 30, 213-218.

Ustinov, Y., Lichstein, K. L., Vander Wal, G. S., Taylor, D. J., Riedel, B. W., \& Bush, A. J. (2010). Association between report of insomnia and daytime functioning. Sleep Medicine, 11, 65-68.

Van Horn, M. L., Jaki, T., Masyn, K., Howe, G., Feaster, D. J., Lamont, A. E., ... Kim, M. (2015). Evaluating differential effects using regression interactions and regression mixture models. Educational and Psychological Measurement, 75, 677-714.

Van Horn, M. L., Smith, J., Fagan, A. A., Jaki, T., Feaster, D. J., Masyn, K., . . . Howe, G. (2012). Not quite normal: Consequences of violating the assumption of normality in regression mixture models. Structural Equation Modeling, 19, 227-249. https://doi.org/10. 1080/10705511.2012.65962

Vitale, J. A., Roveda, E., Montaruli, A., Galasso, L., Weydahl, A., Caumo, A., \& Carandente, F. (2014). Chronotype influences activity circadian rhythm and sleep: Differences in sleep quality between weekdays and weekend. Chronobiology International, 32, 405415. https://doi.org/10.3109/07420528.2014.986273

Wong, Y. J., Owen, J., \& Shea, M. (2012). A latent class regression analysis of men's conformity to masculine norms and psychological distress. Journal of Counseling Psychology, 59, 176-183. https:// doi.org/10.1037/a0026206

Xu, W., \& Hedeker, D. (2001). A random-effects mixture model for classifying treatment response in longitudinal clinical trials. Journal of Biopharmaceutical Statistics, 11, 253-273.

Yau, K. K., Lee, A. H., \& Ng, A. S. (2003). Finite mixture regression model with random effects: Application to neonatal hospital length of stay. Computational Statistics and Data Analysis, 41, 359-366.

Publisher's note Springer Nature remains neutral with regard to jurisdictional claims in published maps and institutional affiliations. 